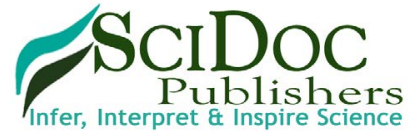

\section{Identifying Surgery and Anesthesia - Related Anxiety in Patients Undergoing Colorectal Cancer Surgery}

\author{
Research Article
}

Lafci $\mathrm{A}^{1 *}$, Gokcinar $\mathrm{D}^{1}$, Dag $\mathrm{O}^{2}$

${ }^{1}$ Department of Anesthesiology, Ankara Numune Training and Research Hospital, Ankara, Turkey.

${ }^{2}$ Department of Biostatistics, Hacettepe University Faculty of Medicine, Ankara, Turkey.

\title{
Abstract
}

Objective: In patients where colorectal cancer surgery is planned, anxiety is triggered not only by cancer diagnosis, but also potentially by the surgery and anesthesia preoperatively. We aimed to determine preoperative anxiety level, identify its reasons, and investigate patients' needs about psychological support.

Methods: Upon approval by ethics committee, this cross-sectional survey study is performed with patients who would undergo colorectal cancer surgery in colorectal surgery department of a tertiary care hospital. Amsterdam Preoperative Anxiety and Information Scale (APAIS), and The State Trait Anxiety Inventory (STAI), and anxiety scores of patients were assessed by a questionnaire.

Results: A total of 110 patients were interviewed. APAIS, S-STAI, and T-STAI were significantly higher in $<60$-year-old patients $(\mathrm{p}<0.001)$. Female patients had statistically higher APAIS $(\mathrm{p}=0.032)$, S-STAI $(\mathrm{p}<0.001)$, and T-STAI $(\mathrm{p}=0.002)$ scores compared with male patients. Regression model showed significantly independent associations of anxiety scores to age, gender, education status, and marital status subgroups.

Conclusion: Among patients, where colorectal cancer surgery was planned, anxiety level was elevated in those below 60 years old, with higher level of education, and female gender. Developing new protocols to reduce stress in these groups may improve perioperative outcomes.

Keywords: Amsterdam Preoperative Anxiety and Information Scale; State Trait Anxiety Inventory; Colorectal Cancer Surgery; Anxiety.

Abbreviations: APAIS: Amsterdam Preoperative Anxiety and Information Scale; STAI: State Trait Anxiety Inventory; ERAS: Enhanced Recovery After Surgery.

\section{Introduction}

Colorectal cancer surgery is a major procedure that requires careful preoperative preparation. It may be associated with significant perioperative complications which increase morbidity and mortality. Appropriate preoperative arrangements provide both reduced complication rates and rapid recovery in postoperative period. Enhanced Recovery After Surgery (ERAS) protocols designated for colorectal surgery include preoperative counselling, absence of mechanical bowel preparation, shortening of preoperative fasting period, carbohydrate loading before the surgery, anesthetic premedication, and antibiotic and thromboembolic prophylaxis.
ERAS protocol was shown to accelerate recovery and significantly shorten length of hospital stay [1-5].

Patients to whom colorectal cancer surgery is planned may have anxiety due to not only the cancer diagnosis itself, but also the surgery and anesthesia. In studies where anesthesia associated fears of surgical patients were investigated, fears about awareness during the surgery, failure of awakening after the surgery, and feeling of pain was reported $[6,7]$. Measuring anxiety and identifying its reasons may help to reduce concerns and fears emerged during preoperative period. Amsterdam Preoperative Anxiety and Information Scale (APAIS) and The State Trait Anxiety Inventory

\section{*Corresponding Author:}

Ayse Lafci,

Department of Anesthesiology, Ankara Numune Egitim ve Arastirma Hastanesi, Talatpaşa Bulvari, No:44, 06100, Altindag, Ankara, Turkey.

Tel: +905052788095

Fax: +903123114340

E-mail: ayselafci971@gmail.com

Received: November 02, 2018

Accepted: December 13, 2018

Published: December 14, 2018

Citation: Lafci A, Gokcinar D, Dag O. Identifying Surgery and Anesthesia - Related Anxiety in Patients Undergoing Colorectal Cancer Surgery. Int J Anesth Res. 2018;6(9):550-554. doi: http://dx.doi.org/10.19070/2332-2780-18000111

Copyright: Lafci $\mathbf{A}^{\odot}$ 2018. This is an open-access article distributed under the terms of the Creative Commons Attribution License, which permits unrestricted use, distribution and reproduction in any medium, provided the original author and source are credited. 
(STAI) were among commonly used tools to determine anxiety level [8-10].

Limited studies exist regarding the need for psychological support to patients in whom colorectal cancer surgery is planned. The aim of this study was to investigate preoperative anxiety level induced by surgery and anesthesia in colorectal cancer surgery patients.

\section{Methods}

This cross-sectional survey study was performed in patients $>$ 18-year-old patients who were planned to have colorectal cancer surgery. It conducted in colorectal surgery department of a tertiary-level hospital upon approval by institutional ethics committee between 1 April 2017 and 30 June 2017. Informed consent form was obtained from all patients. Patients enrolled to the study were questioned about their age, gender, level of education, marital status, and previous surgery. Their anxiety scores were measured with the use of APAIS, S-STAI (state), and T-STAI (trait) scales on the day before surgery. For APAIS, each question is rated on a five-point Likert's scale, where a value of 1 indicates "not anxious at" all and 5 means "extremely anxious" [10]. In response to S-STAI, the patients choose the number that best describes the intensity of their feelings: 1 means "not at all", 2 means "somewhat", 3 means "moderately", 4 means "very much so". In response to T-STAI, patients rate the frequency of their feelings on the following four-point scale: 1 means "almost never", 2 means "sometimes", 3 means "often", 4 means "almost always". Scores for both S-STAI and T-STAI can vary from a minimum of 20 to a maximum of 80 . The high values in APAIS, S-STAI, and T-STAI correspond to high levels of anxiety [9]. Those having history of mental disorder or psychiatric medication use were excluded from the study.

\section{Statistical Analysis}

Data were analyzed using IBM SPSS Statistics for Windows v.23.0 (IBM Corp., Armonk, NY). Descriptive statistics for continu- ous variables were reported as mean \pm standard deviation (median, minimum - maximum). The sample size in each group was stated as frequency and percentage. Shapiro-Wilk normality test was utilized to assess the normality of each continuous variable. Levene-test was used to check the homogeneity of variance. Mann-Whitney $U$ test was applied to compare two groups since these assumptions were not satisfied. Multiple linear regression model was constructed to investigate the individual effect of an independent variable on APAIS, S-STAI and T-STAI by controlling other independent variables. A two-sided P-value $\leq 0.05$ was considered statistically significant.

\section{Results}

A total of 110 patients were questioned in the study. Patients' characteristics were presented at Table 1 . While $47 \%$ of patients were between $60-69$-year age group, $88 \%$ of the population constituted male patients. More than half of patients $(57 \%)$ were primary school graduates, followed by high school (31\%) and university $(12 \%)$ graduates. Most of the responders (84\%) were married. Those having previous surgery other than current surgery constituted $81 \%$ of the population.

As demonstrated at Table 2, APAIS score was found to be significantly higher in $<60$-year-old patients (median: 15 , range: 7-18) compared with that of $\geq 60$-year-old patients (median: 11 , range: 5-19), $(\mathrm{p}<0.001)$. S-STAI $(\mathrm{p}<0.001)$ and T-STAI $(\mathrm{P}<0.001)$ were also statistically higher in $<60$-year-old patients compared with those of $\geq 60$-year-old patients. In female patients, S-STAI score was significantly higher than that of their male counterparts $(p<0.001)$. Regression model indicated that APAIS score was significantly different between $<60$-year age and $\geq 60$-year age groups, independent of other parameters (95\% CI 0.544-3.289, $\mathrm{p}=0.007)$. APAIS, S-STAI, and T-STAI scores were significantly different in terms of gender, education status, and marital status subgroups (Table 3).

When APAIS, S-STAI, and T-STAI scores were compared ac-

Table 1. Baseline characteristics.

\begin{tabular}{|c|c|c|}
\hline \multicolumn{2}{|c|}{} & $\mathrm{n}(\%)$ \\
\hline \multirow{4}{*}{ Age (years) } & $30-39$ & $2(2)$ \\
& $40-49$ & $10(9)$ \\
& $50-59$ & $28(25)$ \\
& $60-69$ & $52(47)$ \\
& $70-79$ & $18(16)$ \\
\hline \multirow{2}{*}{ Gender } & Female & $13(12)$ \\
& Male & $97(88)$ \\
\hline \multirow{3}{*}{ Level of education } & Primary & $63(57)$ \\
& High school & $34(31)$ \\
& Undergraduate & $13(12)$ \\
\hline \multirow{3}{*}{ Marital status } & Single & $3(3)$ \\
& Married & $92(84)$ \\
& Widow & $6(5)$ \\
& Divorced & $9(8)$ \\
\hline \multirow{2}{*}{ Previous surgery } & Yes & $89(81)$ \\
& No & $21(19)$ \\
\hline
\end{tabular}

Note: Results are shown as frequency and percentage. 
Table 2. Comparison of baseline characteristics in terms of anxiety scores.

\begin{tabular}{|c|c|c|c|c|c|c|}
\hline & APAIS & $\mathbf{P}$ & S-STAI & $\mathbf{P}$ & T-STAI & $\mathbf{P}$ \\
\hline \multicolumn{7}{|l|}{ Age } \\
\hline$<60$ years & $14.7 \pm 2.8(15,7-18)$ & \multirow{2}{*}{$<0.001$} & $47.8 \pm 7.3(49,32-58)$ & \multirow{2}{*}{$<0.001$} & $49.7 \pm 6.7(52,36-59)$ & \multirow{2}{*}{$<0.001$} \\
\hline$\geq 60$ years & $11.4 \pm 3.5(11,5-19)$ & & $40.6 \pm 6.7(40,30-55)$ & & $43.4 \pm 6.6(43,33-59)$ & \\
\hline \multicolumn{7}{|l|}{ Gender } \\
\hline Male & $12.3 \pm 3.6(12,5-19)$ & \multirow{2}{*}{0.032} & $42.3 \pm 7.6(40,30-58)$ & \multirow{2}{*}{$<0.001$} & $44.9 \pm 7.3(43,33-59)$ & \multirow{2}{*}{0.002} \\
\hline Female & $14.6 \pm 2.8(14,11-19)$ & & $50.5 \pm 3.0(50,43-55)$ & & $51.8 \pm 2.4(52,44-53)$ & \\
\hline \multicolumn{7}{|l|}{ School } \\
\hline Primary & $11.0 \pm 3.3(11,5-19)$ & \multirow{2}{*}{$<0.001$} & $38.9 \pm 5.5(39,30-52)$ & \multirow{2}{*}{$<0.001$} & $41.8 \pm 5.5(42,33-55)$ & \multirow{2}{*}{$<0.001$} \\
\hline Others & $14.8 \pm 2.7(15,6-19)$ & & $49.0 \pm 6.4(50,30-58)$ & & $50.8 \pm 6.2(52,33-59)$ & \\
\hline \multicolumn{7}{|c|}{ Marital status } \\
\hline Married & $12.3 \pm 3.6(12,5-19)$ & \multirow{2}{*}{0.041} & $42.8 \pm 7.7(41,30-58)$ & \multirow{2}{*}{0.183} & $45.3 \pm 7.3(43,33-59)$ & \multirow{2}{*}{0.269} \\
\hline Others & $14.1 \pm 3.3(15,6-18)$ & & $45.4 \pm 7.4(45,30-56)$ & & $47.6 \pm 7.2(50,33-59)$ & \\
\hline \multicolumn{7}{|c|}{ Previous surgery } \\
\hline Yes & $12.3 \pm 3.6(12,5-18)$ & \multirow{2}{*}{0.055} & $42.9 \pm 7.7(41,30-58)$ & \multirow{2}{*}{0.398} & $45.2 \pm 7.4(44,33-59)$ & \multirow{2}{*}{0.323} \\
\hline No & $14.0 \pm 3.2(14,7-19)$ & & $44.5 \pm 7.7(45,32-53)$ & & $47.6 \pm 6.4(50,36-55)$ & \\
\hline
\end{tabular}

Note: Results are shown as mean \pm standard deviation (median, minimum - maximum). P-values are obtained via Mann-Whitney $U$ test.

Table 3. Predictive factors for APAIS, S-STAI, and T-STAI.

\begin{tabular}{|c|c|c|c|c|}
\hline & \multicolumn{4}{|c|}{$95 \% \mathrm{CI}$} \\
\hline & Coefficient & Lower & Upper & $\mathbf{P}$ \\
\hline \multicolumn{5}{|l|}{ APAIS } \\
\hline Constant & 17.636 & 15.086 & 20.187 & $<0.001$ \\
\hline Age $(<60$ vs. $\geq 60)$ & 1.917 & 0.544 & 3.289 & 0.007 \\
\hline Gender (male vs. female) & -2.145 & -3.930 & -0.359 & 0.019 \\
\hline School (primary vs. others) & -2.507 & -3.841 & -1.174 & $<0.001$ \\
\hline Marital status (married vs. others) & -1.939 & -3.437 & -0.442 & 0.012 \\
\hline Previous surgery (yes vs. no) & -0.981 & -2.400 & 0.439 & 0.174 \\
\hline \multicolumn{5}{|c|}{$n=110, R^{2}=0.630, p$-value $<0.001$} \\
\hline \multicolumn{5}{|l|}{ S-STAI } \\
\hline Constant & 54.016 & 49.248 & 58.785 & $<0.001$ \\
\hline Age (<60 vs. $\geq 60)$ & 3.987 & 1.421 & 6.553 & 0.003 \\
\hline Gender (male vs. female) & -7.194 & -10.533 & -3.856 & $<0.001$ \\
\hline School (primary vs. others) & -7.504 & -9.997 & -5.011 & $<0.001$ \\
\hline Marital status (married vs. others) & -2.733 & -5.534 & 0.067 & 0.056 \\
\hline Previous surgery (yes vs. no) & 0.863 & -1.791 & 3.517 & 0.520 \\
\hline \multicolumn{5}{|c|}{$n=110, \mathrm{R}^{2}=0.738, p$-value $<0.001$} \\
\hline \multicolumn{5}{|l|}{ T-STAI } \\
\hline Constant & 55.872 & 51.028 & 60.717 & $<0.001$ \\
\hline Age $(<60$ vs. $\geq 60)$ & 3.299 & 0.692 & 5.906 & 0.014 \\
\hline Gender (male vs. female) & -5.953 & -9.345 & -2.562 & 0.001 \\
\hline School (primary vs. others) & -6.619 & -9.152 & -4.086 & $<0.001$ \\
\hline Marital status (married vs. others) & -2.525 & -5.370 & 0.321 & 0.081 \\
\hline Previous surgery (yes vs. no) & -0.316 & -3.013 & 2.381 & 0.817 \\
\hline \multicolumn{5}{|c|}{$n=110, \mathrm{R}^{2}=0.685, p$-value $<0.001$} \\
\hline
\end{tabular}


cording to the level of education, high school and university graduates had higher scores compared with primary school graduates $(\mathrm{p}<0.001)$.

APAIS, S-STAI, and T-STAI scores did not significantly differ in terms of either presence of previous surgery or marital status.

\section{Discussion}

APAIS, S-STAI, and T-STAI scores that we investigated to determine anxiety levels in patients who were planned to have colorectal cancer surgery were elevated in $<60$-year-old patients and those with higher education status. S-STAI and T-STAI scores were higher in women. Increased scores in preoperative period indicated mounted anxiety and stress. In fact, fear of surgery may elicit anxiety and stress. ERAS protocol was described to improve perioperative healing in patients where colorectal cancer surgery is intended. Patient counselling on the surgery and anesthesia method during preoperative period and use of premedication is included in the protocol [1-3]. However, there exists no tool for determining anxiety level and corresponding management assigned to this protocol. Identifying whether or not there is a fear of intended colorectal surgery is of paramount importance especially in cancer patients. Recent advances in oncology improved survival for patients. Nevertheless, the damage induced by the cancer may increase as the life span prolongs. In addition, organ functions and quality of life may be impaired by certain factors such as chemotherapy, radiotherapy, hormonotherapy, surgery, and anesthesia [11-13]. Beside physical problems, cancer patients also face problems, including poor communication with physicians and lack of adequate information about their disease, which might have negative impact on healthcare and outcomes $[14,15]$.

Lichtor et al., in their study performed with 52 patients, suggested that age did not predict preoperative anxiety levels [16]. On the contrary, later large-scale studies reported higher incidence of preoperative anxiety in young patients compared to their elderly counterparts $[17,18]$. In a meta-analysis, anxiety disorders were less likely to be seen in $>60$-year-old patients compared to $>60$-year-old patients [19]. Consistent with these findings, we also found lower frequency of anxiety in colorectal cancer surgery patients above 60 years old.

Many studies reported higher incidence of surgery and anesthesia - related fear and anxiety among women [17-21]. In the study by Ruhaiyem et al., where 152 female patients were compared 179 male patients, it was reported that gender was the most important determinant of surgery-related fear, indicating a five-fold elevated fear in women [21]. In our study, anxiety scores were statistically higher in female patients compared with that of male patients. Moreover, regression analysis showed an independent effect of being woman in patients with higher levels of anxiety.

The association between education level and anxiety is controversial. Some studies reported positive correlation of education level and anxiety $[18,22,23]$. We also found a positive association between education level and anxiety. On the other hand, there are also some research reporting negative association between these two parameters [24, 25]. Patients with higher education status question about the surgery, anesthesia, and their complications, and try to be informed. In fact, having adequate knowledge may decrease their anxiety. However, having detailed information may also increase anxiety paradoxically.

A previous study reported no significant association between marital status and preoperative APAIS score [26]. Consistently, we also detected no statistically significant association between marital status and APAIS, S-STAI, and T-STAI scores.

Many studies reported that previous experience of surgery diminished level of preoperative anxiety [10, 19, 22-25, 27]. Contrarily, Saini et al., recently revealed no impact of prior surgical experience on preoperative anxiety [28]. In our study, we also did not detect any reduction in preoperative anxiety by past surgical experience. This may be explained by our selection of study population, consisting of merely colorectal cancer surgery. Studies suggesting diminished preoperative anxiety by past surgical experience mostly included heterogeneous types of surgery. In a study reporting varying levels of preoperative anxiety based on the type of intervention, esthetic, gynecological, and general surgery procedures were shown to cause higher level of anxiety compared with those seen in neurosurgery, cardiovascular surgery, orthopedic surgery, and urological surgery [17]. Accordingly, no reduction in anxiety by past surgical experience in our study may be attributed to the fact that colorectal cancer surgery is also a major procedure.

Limitation of this study is that it is conducted in one center and with a small number of participants. Moreover, other scales could be used to measure stress and anxiety but not used. Future development of specific scales to assess perioperative psychological status and evaluation of patients with large-scale multicentre studies is necessary.

\section{Conclusions}

Being $<60$-year-old or female, or higher education status were found to be associated with elevated anxiety levels among patients where colorectal cancer surgery was intended. In these patients with elevated level of anxiety, it is critical to decrease preoperative stress and fear. New protocols addressing relief of anxiety based on age groups, gender, and education status are warranted in patients with colorectal cancer. This may provide optimal perioperative conditions by reducing patients' stress. Incorporation of parameters targeted to promote psychological support into ERAS may improve mental well-being in addition to priming physiological healing.

\section{References}

[1]. Fearon KC, Ljungqvist O, Von Meyenfeldt M, Revhaug A, Dejong CH, Lassen K, et al. Enhanced recovery after surgery: a consensus review of clinical care for patients undergoing colonic resection. Clin Nutr. 2005 Jun;24(3):466-77. PubMed: 15896435.

[2]. Melnyk M, Casey RG, Black P, Koupparis AJ. Enhanced recovery after surgery (ERAS) protocols: Time to change practice?. Can Urol Assoc J. 2011 Oct;5(5):342-8. doi: 10.5489/cuaj.11002. PubMed PMID: 22031616.

[3]. Basse L, Raskov HH, Jakobsen DH, Sonne E, Billesbølle P, Hendel HW, et al. Accelerated postoperative recovery programme after colonic resection improves physical performance, pulmonary function and body composition. Br J Surg. 2002 Apr;89(4):446-53. PubMed PMID: 11952586.

[4]. Wind J, Polle SW, Jin PF, Dejong CH, Von Meyenfeldt MF, Ubbink DT, et al. Systematic review of enhanced recovery programmes in colonic surgery. Br J Surg. 2006 Jul;93(7):800-9. PubMed PMID: 16775831.

[5]. Kehlet H. Fast-track colorectal surgery. Lancet. 2008 Mar 8;371(9615):7913. doi: 10.1016/S0140-6736(08)60357-8. PubMed PMID: 18328911.

[6]. Stoelting RK, Miller RD. Psychological preparation and preoperative medi- 
cation. Anesth. 1986;1:381-97.

[7]. Gaskey NJ. Evaluation of the effect of a pre-operative anesthesia videotape. AANA J. 1987 Aug;55(4):341-5. PubMed PMID: 3661082.

[8]. VanDyke MM, Parker JC, Smarr KL, Hewett JE, Johnson GE, Slaughter JR, et al. Anxiety in rheumatoid arthritis. Arthritis Rheum. 2004 Jun 15;51(3):408-12. PubMed PMID: 15188326

[9]. Spielberger CD, Gorsuch RL, Lushene R, Vagg PR, Jacobs GA. Manual for the State-Trait Anxiety Inventory. CA: Consulting Psychologists Press; 1983 Jan.

[10]. Moerman N, van Dam FS, Muller MJ, Oosting H. The Amsterdam preoperative anxiety and information scale (APAIS). Anesth Analg. 1996 Mar;82(3):445-51. PubMed PMID: 8623940.

[11]. Jemal A, Clegg LX, Ward E, Ries LA, Wu X, Jamison PM, et al. Annual report to the nation on the status of cancer, 1975-2001, with a special feature regarding survival. Cancer. 2004 Jul 1;101(1):3-27. PubMed PMID: 15221985 .

[12]. Aziz NM, Rowland JH. Trends and advances in cancer survivorship research: challenge and opportunity. Semin Radiat Oncol. 2003 Jul;13(3):248-66. PubMed PMID: 12903014.

[13]. Oeffinger KC, Hudson MM. Long-term complications following childhood and adolescent cancer: Foundations for providing risk-based health care for survivors. CA Cancer J Clin. 2004 Jul-Aug;54(4):208-36. PubMed PMID: 15253918.

[14]. Eakin EG, Strycker LA. Awareness and barriers to use of cancer support and information resources by HMO patients with breast, prostate, or colon cancer: patient and provider perspectives. Psychooncology. $2001 \mathrm{Mar}-$ Apr;10(2):103-13. PubMed PMID: 11268137.

[15]. Skalla KA, Bakitas M, Furstenberg CT, Ahles T, Henderson JV. Patients' need for information about cancer therapy. Oncol Nurs Forum. 2004 MarApr;31(2):313-9. PubMed PMID: 15017447.

[16]. Lichtor JL, Johanson CE, Mhoon D, Faure EA, Hassan SZ, Roizen MF. Preoperative Anxiety Does Anxiety Level the Afternoon Before Surgery Predict Anxiety Level Just Before Surgery?. Anesthesiology. 1987 Oct;67(4):595-9. PubMed PMID: 3662091.

[17]. Erkilic E, Kesimci E, Soykut C, Doger C, Gumus T, Kanbak O. Factors as- sociated with preoperative anxiety levels of Turkish surgical patients: from a single center in Ankara. Patient Prefer Adherence. 2017 Feb 28;11:291-296. doi: 10.2147/PPA.S127342. PubMed PMID: 28280304.

[18]. Jafar MF, Khan FA. Frequency of preoperative anxiety in Pakistani surgical patients. J Pak Med Assoc. 2009 Jun;59(6):359-63. PubMed PMID: 19534368.

[19]. Flint AJ. Epidemiology and comorbidity of anxiety disorders in the elderly. Am J Psychiatry. 1994 May;151(5):640-9. PubMed PMID: 8166303.

[20]. Matthias AT, Samarasekera DN. Preoperative anxiety in surgical patientsexperience of a single unit. Acta Anaesthesiol Taiwan. 2012 Mar;50(1):3-6. doi: 10.1016/j.aat.2012.02.004. PubMed PMID: 22500906

[21]. Ruhaiyem ME, Alshehri AA, Saade M, Shoabi TA, Zahoor H, Tawfeeq NA. Fear of going under general anesthesia: A cross-sectional study. Saudi J Anaesth. 2016 Jul-Sep;10(3):317-21. doi: 10.4103/1658-354X.179094. PubMed PMID: 27375388.

[22]. Domar AD, Everett LL, Keller MG. Preoperative anxiety: is it a predictable entity?. Anesth Analg. 1989 Dec;69(6):763-7. PubMed PMID: 2589657.

[23]. Caumo W, Schmidt AP, Schneider CN, Bergmann J, Iwamoto CW, Bandeira $\mathrm{D}$, et al. Risk factors for preoperative anxiety in adults. Acta Anaesthesiol Scand. 2001 Mar;45(3):298-307. PubMed PMID: 11207465.

[24]. Kindler CH, Harms C, Amsler F, Ihde-Scholl T, Scheidegger D. The visual analog scale allows effective measurement of preoperative anxiety and detection of patients' anesthetic concerns. Anesth Analg. 2000 Mar;90(3):706-12. PubMed PMID: 10702461.

[25]. Uddin I, Kurkuman AR, Jamil T. Pre-operative anxiety in patients admitted for elective surgery in King Saud Hospital, Unaizah, Al-Qassim, Kingdom of Saudi Arabia. Pak J Med Sci. 2002;18(4):306-10.

[26]. Sirinan C, Rungreungvanich M, Vijitpavan A, Morkchareonpong C. Preanesthetic anxiety assessment: HADS versus APAIS. Thailand J Anesth. 2000;26:155-63.

[27]. Badner NH, Nielson WR, Munk S, Kwiatkowska C, Gelb AW. Preoperative anxiety: detection and contributing factors. Can J Anaesth. 1990 May;37(4 Pt 1):444-7. PubMed PMID: 2340614.

[28]. Saini S, Dayal M. Preoperative Anxiety in Indian Surgical Patients-Experience of a Single Unit. Indian J Appl Res. 2016;6(9):476-9. 